IJMMS 32:5 (2002) 263-270

PII. S0161171202109045

http://ijmms.hindawi.com

(c) Hindawi Publishing Corp.

\title{
FUZZY POSITIVE IMPLICATIVE ORDERED FILTERS OF IMPLICATIVE SEMIGROUPS
}

\author{
YOUNG BAE JUN, YOUNG HEE KIM, and HEE SIK KIM
}

Received 11 September 2001 and in revised form 5 May 2002

\begin{abstract}
We consider the fuzzification of the notion of a positive implicative ordered filter in implicative semigroups. We show that every fuzzy positive implicative ordered filter is both a fuzzy ordered filter and a fuzzy implicative ordered filter. We give examples that a fuzzy (implicative) ordered filter may not be a fuzzy positive implicative ordered filter. We also state equivalent conditions of fuzzy positive implicative ordered filters. Finally, we establish an extension property for fuzzy positive implicative ordered filters.
\end{abstract}

2000 Mathematics Subject Classification: 20M12, 06F05, 03B52.

1. Introduction. The notions of implicative semigroup and ordered filter were introduced by Chan and Shum [3]. The first is a generalization of implicative semilattice (see Nemitz [10] and Blyth [2]) and has a close relation to implication in mathematical logic and set theoretic difference (see Birkhoff [1] and Curry [4]). For the general development of implicative semilattice theory, the ordered filters play an important role which is shown by Nemitz [10]. Motivated by this, Chan and Shum [3] established some elementary properties and constructed quotient structure of implicative semigroups via ordered filters. Jun et al. [8] discussed ordered filters of implicative semigroups. Also, Jun [6] studied implicative ordered filters of implicative semigroups. Jun and Kim [7] introduced the notion of positive implicative ordered filters in implicative semigroups and gave relations between (implicative) ordered filters and positive implicative ordered filters. On the other hand, Jun, together with Kuresh and Huang, considered the fuzzification of the (implicative) ordered filter of implicative semigroups (see $[5,9])$. In this paper, we introduce the notion of fuzzy positive implicative ordered filters in implicative semigroups and give relations between fuzzy (implicative) ordered filters and fuzzy positive implicative ordered filters. We provide characterizations of fuzzy positive implicative ordered filters and establish an extension property for fuzzy positive implicative ordered filters.

2. Preliminaries. We recall some definitions and results. By a negatively partially ordered semigroup (n.p.o. semigroup), we mean a set $S$ with a partial ordering " $\leq$ " and a binary operation "." such that for all $x, y, z \in S$, we have the following:

(i) $(x \cdot y) \cdot z=x \cdot(y \cdot z)$,

(ii) $x \leq y$ implies $x \cdot z \leq y \cdot z$ and $z \cdot x \leq z \cdot y$,

(iii) $x \cdot y \leq x$ and $x \cdot y \leq y$. 
An n.p.o. semigroup $(S ; \leq, \cdot)$ is said to be implicative if there is an additional binary operation $*: S \times S \rightarrow S$ such that for any elements $x, y, z$ of $S$,

(iv) $z \leq x * y$ if and only if $z \cdot x \leq y$.

The operation $*$ is called implication. From now on, an implicative n.p.o. semigroup is simply called an implicative semigroup.

An implicative semigroup $(S ; \leq, \cdot, *)$ is said to be commutative if it satisfies

(v) $x \cdot y=y \cdot x$ for all $x, y \in S$, that is, $(S, \cdot)$ is a commutative semigroup.

In any implicative semigroup $(S ; \leq, \cdot, *), x * x=y * y$, and this element is the greatest in $S$; it will be denoted by 1 .

Some elementary properties of implicative semigroups are summarized by the following proposition.

Proposition 2.1 (see [3, Theorem 1.4]). Let $S$ be an implicative semigroup. Then, for every $x, y, z \in S$, the following holds:

(P1) $x \leq 1, x * x=1, x=1 * x$,

(P2) $x \leq y *(x \cdot y)$,

(P3) $x \leq x * x^{2}$,

(P4) $x \leq y * x$,

(P5) if $x \leq y$ then $x * z \geq y * z$ and $z * x \leq z * y$,

(P6) $x \leq y$ if and only if $x * y=1$,

(P7) $x *(y * z)=(x \cdot y) * z$,

(P8) if $S$ is commutative then $x * y \leq(s \cdot x) *(s \cdot y)$ for all $s$ in $S$.

Proposition 2.2 (see [7, Observation 1.5]). If $S$ is a commutative implicative semigroup, then for any $x, y, z \in S$,

(P9) $x *(y * z)=y *(x * z)$,

(P10) $x \leq(x * y) * y$,

(P11) $y * z \leq(z * x) *(y * x)$,

(P12) $y * z \leq(x * y) *(x * z)$,

(P13) $((x * y) * y) * y=x * y$.

DEFINITION 2.3 (see [3, Definition 2.1]). Let $S$ be an implicative semigroup and let $F$ be a nonempty subset of $S$. Then, $F$ is called an ordered filter of $S$ if it satisfies the following conditions:

(F1) $x \cdot y \in F$ for every $x, y \in F$; that is, $F$ is a subsemigroup of $S$;

(F2) if $x \in F$ and $x \leq y$, then $y \in F$.

The following result gives an equivalent condition of an ordered filter.

Proposition 2.4 (see [8, Proposition 2]). Suppose $S$ is an implicative semigroup. Then, a nonempty subset $F$ of $S$ is an ordered filter if and only if it satisfies the following conditions:

(F3) $1 \in F$,

(F4) $x * y \in F$ and $x \in F$ imply $y \in F$.

DEFINITION 2.5 (see [6]). Let $S$ be an implicative semigroup. A nonempty subset $F$ of $S$ is called an implicative ordered filter of $S$ if it satisfies (F3) and for all $x, y, z \in S$,

(F5) $x *(y * z) \in F$ and $x * y \in F$ imply $x * z \in F$. 
Note that every implicative ordered filter is an ordered filter (see [6, Proposition 3.3]).

Definition 2.6 (see [5, 9]). A fuzzy set $\mu$ in an implicative semigroup $S$ is called a fuzzy ordered filter of $S$ if it satisfies the following conditions:

(F6) $\mu(1) \geq \mu(x)$, for all $x \in S$,

(F7) $\mu(y) \geq \min \{\mu(x * y), \mu(x)\}$, for all $x, y \in S$.

A fuzzy set $\mu$ in an implicative semigroup $S$ is called a fuzzy implicative ordered filter of $S$ if it satisfies condition (F6) and

(F8) $\mu(x * z) \geq \min \{\mu(x *(y * z)), \mu(x * y)\}$, for all $x, y \in S$.

Note that every fuzzy ordered filter is order preserving (see [5, Lemma 3.2]), and every fuzzy implicative ordered filter is a fuzzy ordered filter (see [5, Theorem 3.7]).

3. Fuzzy positive implicative ordered filters. In what follows, let $S$ denote an implicative semigroup unless otherwise specified. Jun and Kim [7] introduced the notion of positive implicative ordered filters in implicative semigroups as follows in the paper.

DEFINITION 3.1 (see [7]). A nonempty subset $F$ of $S$ is called a positive implicative ordered filter of $S$ if it satisfies (F3) and for all $x, y, z \in S$,

(F9) $x *((y * z) * y) \in F$ and $x \in F$ imply $y \in F$.

We consider the fuzzification of positive implicative ordered filter as in the following definition.

DEFINITION 3.2. A fuzzy set $\mu$ in $S$ is called a fuzzy positive implicative ordered filter of $S$ if it satisfies condition (F6) and for all $x, y, z \in S$, (F10) $\mu(y) \geq \min \{\mu(x *((y * z) * y)), \mu(x)\}$.

EXAMPLE 3.3. Consider an implicative semigroup $S=\{1, a, b, c, d\}$ with Cayley tables (Tables 3.1 and 3.2) and Hasse diagram (Figure 3.1) (see [7]).

It is easy to check that a fuzzy set $\mu$ in $S$ defined by $\mu(1)=\mu(a)=\mu(b)=0.6$ and $\mu(c)=\mu(d)=0.2$ is a fuzzy positive implicative ordered filter of $S$.

THEOREM 3.4. Every fuzzy positive implicative ordered filter is a fuzzy ordered filter.

Proof. Let $\mu$ be a fuzzy positive implicative ordered filter of $S$ and let $x, y \in S$. Then,

$$
\begin{aligned}
\mu(y) & \geq \min \{\mu(x *((y * y) * y)), \mu(x)\}, \quad \text { by }(\mathrm{F} 10) \\
& =\min \{\mu(x * y), \mu(x)\}, \quad \text { by }(\mathrm{P} 1)
\end{aligned}
$$

and so $\mu$ is a fuzzy ordered filter of $S$.

LEMMA 3.5 (see [5, Theorem 3.3]). Let $\mu$ be a fuzzy set in a commutative implicative semigroup $S$. Then $\mu$ is a fuzzy ordered filter of $S$ if and only if $x \cdot y \leq z$ implies $\mu(z) \geq \min \{\mu(x), \mu(y)\}$. 
TABLE 3.1

\begin{tabular}{c|lllll}
\hline$\cdot$ & 1 & $a$ & $b$ & $c$ & $d$ \\
\hline 1 & 1 & $a$ & $b$ & $c$ & $d$ \\
$a$ & $a$ & $a$ & $d$ & $c$ & $d$ \\
$b$ & $b$ & $d$ & $b$ & $d$ & $d$ \\
$c$ & $c$ & $c$ & $d$ & $c$ & $d$ \\
$d$ & $d$ & $d$ & $d$ & $d$ & $d$ \\
\hline
\end{tabular}

TABLE 3.2

\begin{tabular}{l|lllll}
\hline$*$ & 1 & $a$ & $b$ & $c$ & $d$ \\
\hline 1 & 1 & $a$ & $b$ & $c$ & $d$ \\
$a$ & 1 & 1 & $b$ & $c$ & $d$ \\
$b$ & 1 & $a$ & 1 & $c$ & $c$ \\
$c$ & 1 & 1 & $b$ & 1 & $b$ \\
$d$ & 1 & 1 & 1 & 1 & 1 \\
\hline
\end{tabular}

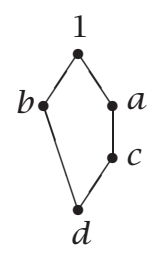

FIGURE 3.1

THEOREM 3.6. Let $S$ be a commutative implicative semigroup. Then, every fuzzy positive implicative ordered filter of $S$ is a fuzzy implicative ordered filter.

Proof. Let $\mu$ be a fuzzy positive implicative ordered filter of $S$ and let $x, y, z \in S$. Since

$$
x *(y * z)=y *(x * z) \leq(x * y) *(x *(x * z))
$$

by (P9) and (P12), we have $(x *(y * z)) \cdot(x * y) \leq x *(x * z)$ using (P7). It follows that

$$
\begin{aligned}
\min \{\mu & (x *(y * z)), \mu(x * y)\} \\
& \leq \mu(x *(x * z)) \quad \text { by Lemma } 3.5 \\
& =\mu(x *(((x * z) * z) * z)) \quad \text { by }(\mathrm{P} 13) \\
& =\mu(((x * z) * z) *(x * z)) \quad \text { by }(\mathrm{P} 9) \\
& =\mu(1 *(((x * z) * z) *(x * z))) \quad \text { by }(\mathrm{P} 1) \\
& =\min \{\mu(1 *(((x * z) * z) *(x * z))), \mu(1)\} \quad \text { by }(\mathrm{F} 6) \\
& \leq \mu(x * z) \quad \text { by }(\mathrm{F} 10) .
\end{aligned}
$$

Hence, $\mu$ is a fuzzy implicative ordered filter of $S$. 
The following example shows that the converses of Theorems 3.4 and 3.6 may not be true.

EXAMPLE 3.7. Let $S$ be the implicative semigroup in Example 3.3. Note that $S$ is commutative. Define a fuzzy set $\mu$ in $S$ by $\mu(1)=\mu(b)=0.8$ and $\mu(a)=\mu(c)=$ $\mu(d)=0.08$. Then, $\mu$ is a fuzzy implicative ordered filter, and hence a fuzzy ordered filter of $S$. But it is not a fuzzy positive implicative ordered filter of $S$, since

$$
\mu(a)=0.08<0.8=\min \{\mu(b *((a * d) * a)), \mu(b)\}
$$

Now we give equivalent conditions for a fuzzy ordered filter (fuzzy implicative ordered filter) to be a fuzzy positive implicative ordered filter.

THEOREM 3.8. Let $\mu$ be a fuzzy ordered filter of $S$. Then, $\mu$ is a fuzzy positive implicative ordered filter of $S$ if and only if

$$
\mu(x) \geq \mu((x * y) * x), \quad \forall x, y \in S .
$$

Proof. Assume that $\mu$ is a fuzzy positive implicative ordered filter of $S$ and let $x, y \in S$. Then

$$
\begin{aligned}
\mu(x) & \geq \min \{\mu(1 *((x * y) * x)), \mu(1)\} \\
& =\mu(1 *((x * y) * x)) \\
& =\mu((x * y) * x) .
\end{aligned}
$$

Conversely, let $\mu$ be a fuzzy ordered filter of $S$ satisfying (3.5). Using (3.5) and (F7), we get

$$
\mu(y) \geq \mu((y * z) * y) \geq \min \{\mu(x *((y * z) * y)), \mu(x)\}
$$

for all $x, y, z \in S$. Hence, $\mu$ is a fuzzy positive implicative ordered filter of $S$.

Proposition 3.9. Let $\mu$ be a fuzzy positive implicative ordered filter of $S$. If $S$ is commutative, then $\mu$ satisfies the following inequality:

$$
\mu((y * x) * x) \geq \mu((x * y) * y), \quad \forall x, y \in S .
$$

Proof. Let $\mu$ be a fuzzy positive implicative ordered filter of a commutative implicative semigroup $S$. Since $x \leq(y * x) * x$ by (P4), it follows from (P5) that

$$
((y * x) * x) * y \leq x * y
$$

Using (P11), (P9), and then (P5) on (3.9), we have

$$
\begin{aligned}
(x * y) * y & \leq(y * x) *((x * y) * x) \\
& =(x * y) *((y * x) * x) \\
& \leq(((y * x) * x) * y) *((y * x) * x) .
\end{aligned}
$$


Since $\mu$ is order preserving, it follows from (F6), (F10), and (P1) that

$$
\begin{aligned}
& \mu((y * x) * x) \\
& \quad \geq \min \{\mu(1 *((((y * x) * x) * y) *((y * x) * x))), \mu(1)\} \\
& \quad=\mu((((y * x) * x) * y) *((y * x) * x)) \\
& \quad \geq \mu((x * y) * y) .
\end{aligned}
$$

This completes the proof.

LEMMA 3.10 (see [5, Lemma 3.14]). Every fuzzy implicative ordered filter $\mu$ of $S$ satisfies the following inequality:

$$
\mu(x * y) \geq \mu(x *(x * y)), \quad \forall x, y \in S .
$$

THEOREM 3.11. If $S$ is commutative, then every fuzzy implicative ordered filter $\mu$ of $S$ satisfying (3.8) is a fuzzy positive implicative ordered filter of $S$.

Proof. In view of Theorem 3.8, it is sufficient to show that $\mu$ satisfies condition (3.5). Note from (P11) that

$$
(x * y) * x \leq(x * y) *((x * y) * y), \quad \forall x, y \in S,
$$

and from (P4) and (P5) that

$$
(x * y) * x \leq y * x, \quad \forall x, y \in S .
$$

Since $\mu$ is order preserving, it follows from (3.8) and Lemma 3.10 that

$$
\begin{aligned}
\mu((y * x) * x) & \geq \mu((x * y) * y) \\
& \geq \mu((x * y) *((x * y) * y)) \\
& \geq \mu((x * y) * x),
\end{aligned}
$$

so that

$$
\begin{aligned}
\mu(x) & \geq \min \{\mu((y * x) * x), \mu(y * x)\} \quad \text { by }(\mathrm{F} 7) \\
& \geq \min \{\mu((x * y) * x), \mu(y * x)\} \\
& =\mu((x * y) * x) \quad \text { by }(3.14) .
\end{aligned}
$$

Hence, $\mu$ is a fuzzy positive implicative ordered filter of $S$.

COROLLARY 3.12. Let $\mu$ be a fuzzy implicative ordered filter of a commutative implicative semigroup $S$. Then, $\mu$ is a fuzzy positive implicative ordered filter of $S$ if and only if it satisfies (3.8).

The following lemmas will be needed in the sequel.

LEMma 3.13 (see [5, Theorem 3.17]). Let $\mu$ and $v$ be fuzzy ordered filters of a commutative implicative semigroup $S$ such that $\mu(1)=v(1)$ and $\mu \leq v$, that is, $\mu(x) \leq v(x)$ for all $x \in S$. If $\mu$ is a fuzzy implicative ordered filter of $S$, then so is $v$. 
LEMMA 3.14 (see [5, Theorem 3.16]). Let $\mu$ be a fuzzy set in a commutative implicative semigroup $S$. Then, $\mu$ is a fuzzy implicative ordered filter of $S$ if and only if $\mu$ is a fuzzy ordered filter of $S$ that satisfies

$$
\mu((x * y) *(x * z)) \geq \mu(x *(y * z)), \quad \forall x, y, z \in S
$$

We finally give an extension property for fuzzy positive implicative ordered filters.

THEOREM 3.15. Let $\mu$ and $v$ be fuzzy ordered filters of a commutative implicative semigroup $S$ such that $\mu(1)=v(1)$ and $\mu \leq v$, that is, $\mu(x) \leq v(x)$ for all $x \in S$. If $\mu$ is a fuzzy positive implicative ordered filter of $S$, then so is $v$.

PROoF. Since every fuzzy positive implicative ordered filter is a fuzzy implicative ordered filter (see Theorem 3.6), it follows from Lemma 3.13 that $v$ is a fuzzy implicative ordered filter of $S$. For any $x, y \in S$, let $a:=(x * y) * y$. Then

$$
\begin{aligned}
v(((a * y) * x) * x) & \geq \mu(((a * y) * x) * x) \\
& \geq \mu((x *(a * y)) *(a * y)) \quad \text { by (3.8) } \\
& =\mu((a *(x * y)) *(a * y)) \quad \text { by (P9) } \\
& \geq \mu(a *((x * y) * y)) \quad \text { by }(3.17) \\
& =\mu(1) \quad \text { by }(\mathrm{P} 1) \\
& =v(1) .
\end{aligned}
$$

On the other hand, using (P10) and (P11) we have

$$
(x * y) * y=a \leq(a * y) * y \leq(((a * y) * x) * x) *((y * x) * x),
$$

and so

$$
v((x * y) * y) \leq v((((a * y) * x) * x) *((y * x) * x))
$$

Applying (3.18), (3.20), (F6), and (F7), we get

$$
\begin{aligned}
v((y * x) * x) & \geq \min \{v((((a * y) * x) * x) *((y * x) * x)), v(((a * y) * x) * x)\} \\
& \geq \min \{v((x * y) * y), v(1)\} \\
& =v((x * y) * y)
\end{aligned}
$$

It follows from Theorem 3.11 that $v$ is a fuzzy positive implicative ordered filter of $S$.

ACKNOWLEDGMents. The authors would like to thank the referees for their valuable suggestions and comments. The first author was supported by Korea Research Foundation grant KRF-2001-005-D00002. 


\section{REFERENCES}

[1] G. Birkhoff, Lattice Theory, 3rd ed., American Mathematical Society Colloquium Publications, vol. 25, American Mathematical Society, Rhode Island, 1967.

[2] T. S. Blyth, Pseudo-residuals in semigroups, J. London Math. Soc. 40 (1965), 441-454.

[3] M. W. Chan and K. P. Shum, Homomorphisms of implicative semigroups, Semigroup Forum 46 (1993), no. 1, 7-15.

[4] H. B. Curry, Foundations of Mathematical Logic, McGraw-Hill, New York, 1963.

[5] Y. B. Jun, Fuzzy implicative ordered filters in implicative semigroups, submitted to Southeast Asian Bull. Math.

[6] _ Implicative ordered filters of implicative semigroups, Commun. Korean Math. Soc. 14 (1999), no. 1, 47-55.

[7] Y. B. Jun and K. H. Kim, Positive implicative ordered filters of implicative semigroups, Int. J. Math. Math. Sci. 23 (2000), no. 12, 801-806.

[8] Y. B. Jun, J. Meng, and X. L. Xin, On ordered filters of implicative semigroups, Semigroup Forum 54 (1997), no. 1, 75-82.

[9] S. W. Kuresh, Y. B. Jun, and W. Huang, Fuzzy ordered filters in implicative semigroups, Chinese Quart. J. Math. 13 (1998), no. 2, 53-57.

[10] W. C. Nemitz, Implicative semi-lattices, Trans. Amer. Math. Soc. 117 (1965), 128-142.

Young Bae Jun: Department of Mathematics Education, Gyeongsang National UNIVERSITY, CHINJU 660-701, KOREA

E-mail address: ybjun@nongae.gsnu.ac.kr

Young HeE Kim: Department of Mathematics, Chungbuk National University, CHONGJU 361-763, KOREA

E-mail address: yhkim@cbucc. chungbuk.ac.kr

Hee Sik Kim: Department of Mathematics, Hanyang University, Seoul 133-791, Korea

E-mail address: heekim@hanyang.ac.kr 


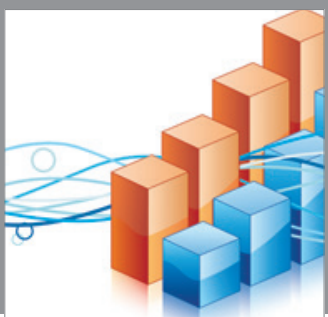

Advances in

Operations Research

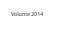

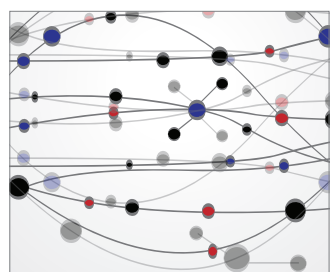

\section{The Scientific} World Journal
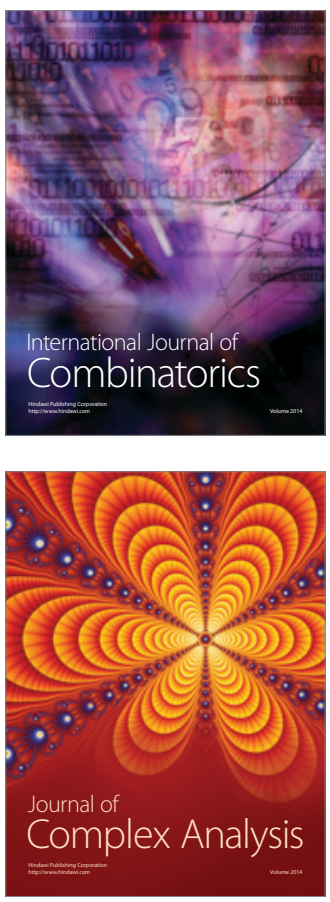

International Journal of

Mathematics and

Mathematical

Sciences
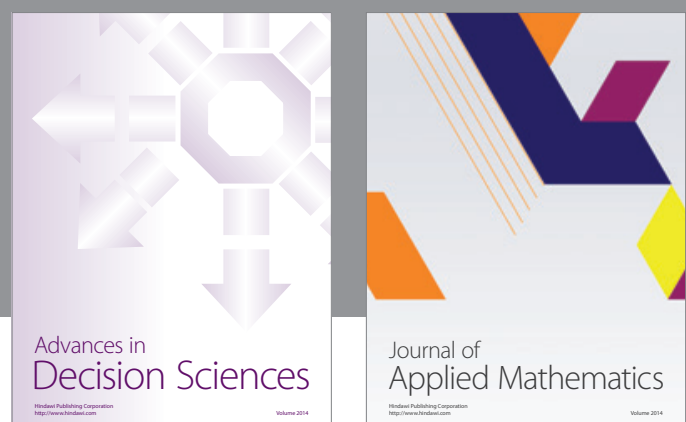

Journal of

Applied Mathematics
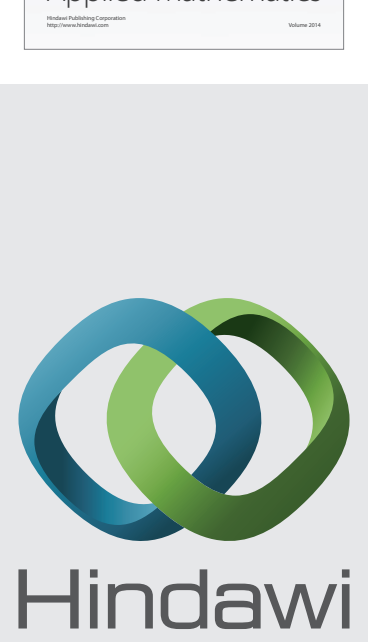

Submit your manuscripts at http://www.hindawi.com
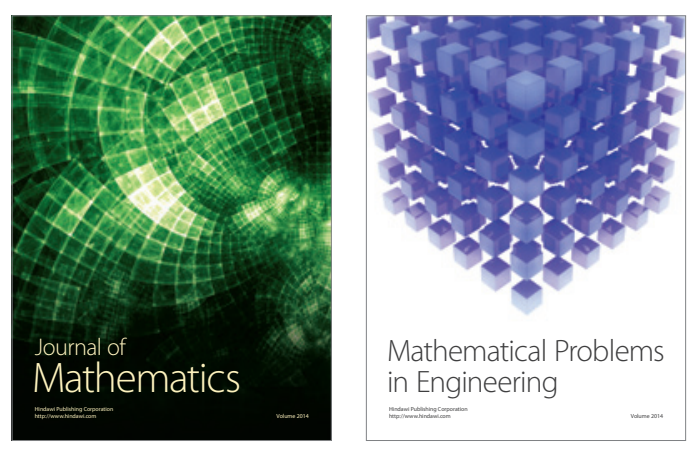

Mathematical Problems in Engineering
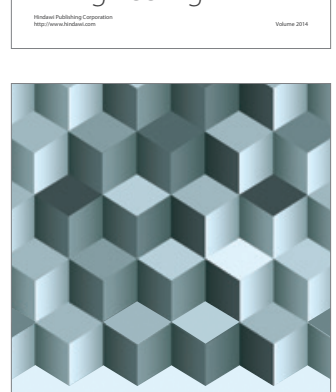

Journal of

Function Spaces
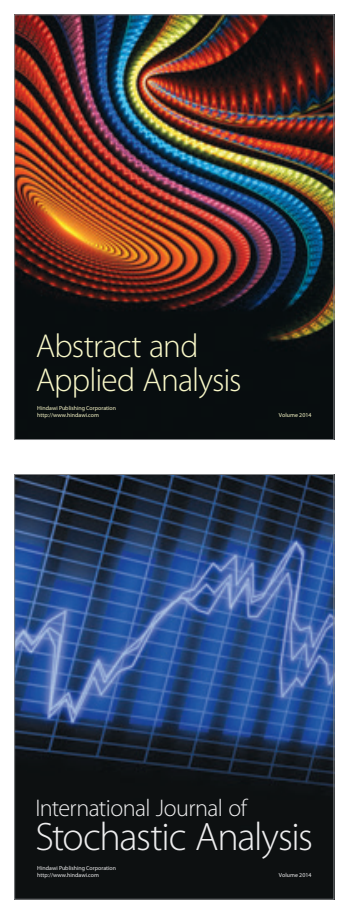

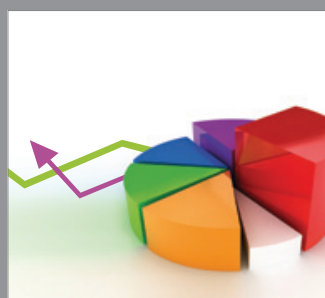

ournal of

Probability and Statistics

Promensencen
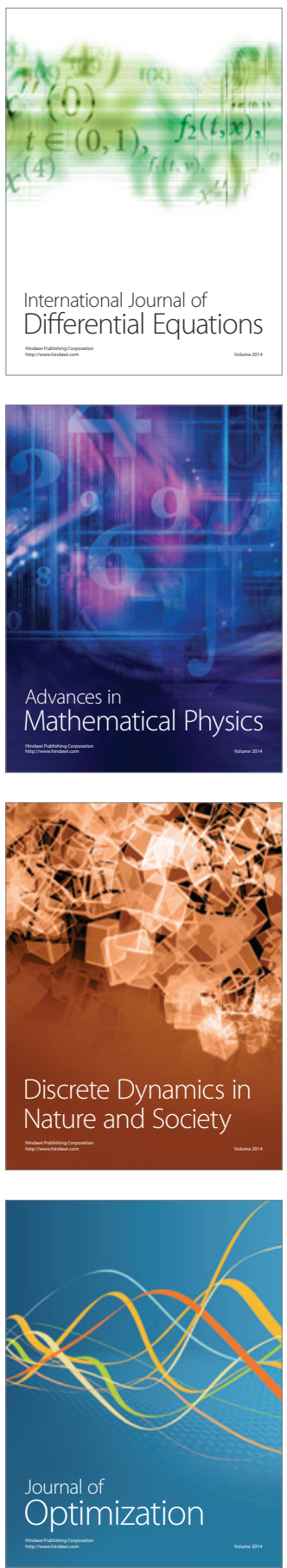